\title{
Investigation on carbon nanomaterials: Coaxial CNT-cylinders and CNT-polymer composite
}

\author{
KALPANA AWASTHI, T P YADAV, P R MISHRA, S AWASTHI and \\ O N SRIVASTAVA* \\ Department of Physics, Banaras Hindu University, Varanasi 221005 , India
}

\begin{abstract}
The macroscopic coaxial carbon cylinders (dia. $\sim 0.5 \mathrm{~cm}$ with varying lengths, $\sim 7-10 \mathrm{~cm}$ ) consisting of aligned carbon nanotube (CNT) stacks have been prepared by controlled spray pyrolysis method. The coaxial carbon cylinders of CNT stacks have been formed directly inside the quartz tube. Another study is done on multi-walled CNTs (MWNTs)-polymer (e.g. polyethylene oxide (PEO), polyacrylamide (PAM)) composite films. We have investigated the structural, electrical and mechanical properties of MWNTs-PEO composites. Composites with different $w \mathrm{t} \%$ (between 0 and $50 \mathrm{wt} \%$ of MWNTs) have been prepared and characterized by the scanning electron microscopic technique. Enhanced electrical conductivity and mechanical strength were observed for the MWNTs-PEO composites. We have also studied the electrical property of MWNTs-PAM composite films.
\end{abstract}

Keywords. Coaxial carbon cylinders; polyethylene oxide; electrical conductivity; mechanical properties.

\section{Introduction}

Since carbon nanotubes (CNTs) were first reported by Iijima in 1991, they soon attracted worldwide interest and have become one of the most promising building blocks for nanotechnology because of their unique structures and excellent electronic, mechanical and chemical properties (Vajtai et al 2002; Meyyappan 2005). Many methods of producing CNTs have been devised including electric arc evaporation, laser evaporation and chemical vapour deposition (CVD) amongst many others (Ebbsen and Ajayan 1992; Thess et al 1996; Zhang et al 2000). However, CVD method (in which hydrocarbon is pyrolyzed in the presence of transition metal catalysts) has attracted a great deal of interest. Several investigations have reported the formation of multiple CNT stack configuration over the patterned substrate by a multibatch CVD process (Singh et al 2003; Vivekchand et al 2004; Li et al 2005; Zhu et al 2005). In our earlier study, we have reported the formation of macroscopic carbon cylinders (diameter, $\sim 0.5 \mathrm{~cm}$ and length, $\sim 5 \mathrm{~cm}$ ) consisting of ordered and aligned CNTs (diameter, $\sim 200-300 \mathrm{~nm}$ and length, $\sim 100 \mu \mathrm{m}$ ) through CVD technique by spraying benzene and ferrocene as a precursor (Srivastava et al 2004). This carbon cylinder was used in the filtration of heavier hydrocarbon species from petroleum and also in the removal of bacteria from the drinking water. For better filtration purposes, probably coaxial carbon cylinders consisting of aligned

*Author for correspondence (hepons@yahoo.com)
CNT stacks may be more useful. This is so since multiple filtering may take place with CNT stacks. In the present investigation, we have prepared coaxial carbon cylinder consisting of aligned CNT stacks by controlled spray pyrolysis method without using any patterned substrate. These coaxial carbon cylinders have been characterized through scanning electron microscopy (SEM) (Philips XL-20) technique.

One class of CNT materials is CNT composites in which the CNTs are embedded within a host matrix material. Polymer composites containing CNTs are of great interest because they may possess a novel combination of electrical, optical and mechanical properties (Moniruzzaman and Winey 2006; Grossiord et al 2006). The effective utilization of CNTs in composite applications depends strongly on the ability to disperse the CNTs homogeneously throughout the matrix without destroying the integrity of the CNTs. Currently, three methods are widely used to incorporate CNTs into polymers: (i) solution mixing or film casting of suspensions of CNTs in dissolved polymer (Kymakis et al 2002), (ii) in situ polymerization of CNT-polymer monomer mixture (Jia et al 1999) and (iii) melt mechanical mixing of CNTs with polymers (Jin et al 2002). Keeping these aspects in view, we have studied the electrical and mechanical properties of CNT-polymer (e.g. polyethylene oxide (PEO), polyacrylamide (PAM)) composites at different CNT concentrations. Among insulating polymers, PEO and PAM have superiority for applications because of its unique properties, such as good strength, stability in air and ease of preparation. The as prepared CNT-polymer (e.g. PEO/PAM) composite films have been characterized by SEM. 


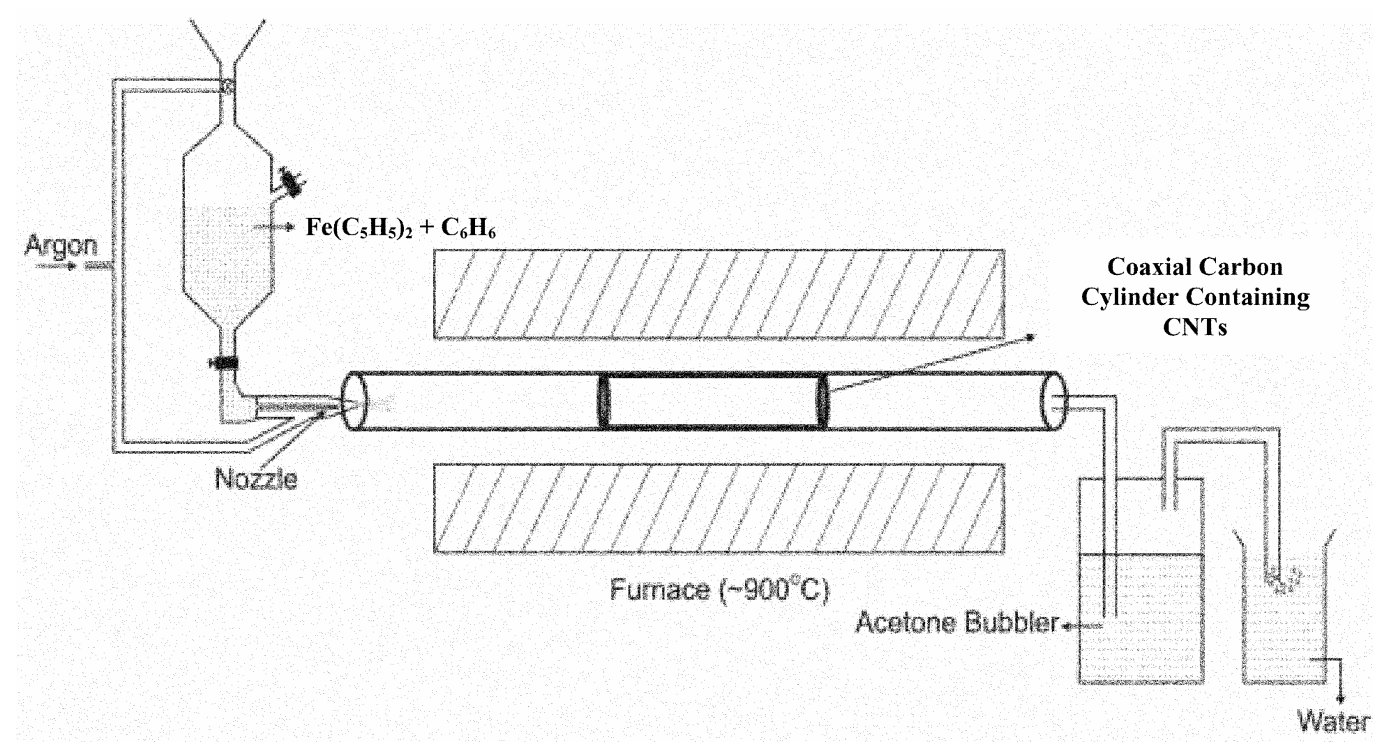

Figure 1. The schematic diagram of spray pyrolysis setup consisting of a pyrex nozzle (i.d = $0.50 \mathrm{~mm})$ used for storing and releasing ferrocene $\left[\mathrm{Fe}\left(\mathrm{C}_{5} \mathrm{H}_{5}\right)_{2}\right]$-benzene $\left(\mathrm{C}_{6} \mathrm{H}_{6}\right)$ solutions.

\section{Experimental}

\subsection{Formation of coaxial carbon cylinder consisting of} aligned CNT stacks

The coaxial carbon cylinders were prepared by spray pyrolysis of benzene-ferrocene solution in argon atmosphere at $\sim 900^{\circ} \mathrm{C}$ temperature. Figure 1 shows schematic illustration of spray pyrolysis setup. Details of the synthesis process of coaxial carbon cylinder have already been described by us (Mishra et al 2007). In order to arrest the growth of the expected aligned tube architecture and to explore the formation of another similar CNT configuration-forming stack, we terminated the flow of precursor solution for a short period ( $\sim 5 \mathrm{~min})$ while keeping the flow of argon uninterrupted. After the interruption, the precursor was sprayed under the same spray conditions i.e. the temperature and flow rate of argon were $\sim 900^{\circ} \mathrm{C}$ and $\sim 5 \mathrm{ml} / \mathrm{min}$, respectively. The as deposited coaxial carbon cylinder was removed from the quartz tube.

\subsection{Synthesis of CNT-polymer composite films}

The CNTs-polymer (e.g. PEO and PAM) composites were prepared by solution cast technique (Awasthi et al 2006). The CNTs were synthesized by the vapour phase pyrolysis of ferrocene along with ethylene $\left(\mathrm{C}_{2} \mathrm{H}_{4}\right)$ (Awasthi et al 2003). The CNTs grown here were multiwalled CNTs (MWNTs). Various mass fraction composites were prepared by blending the PEO/PAM and CNTs in different ratios. The ratio of CNTs in this work corresponds to 5, $10,15,20,30,40$ and $50 \mathrm{wt} \%$ for which films were prepared.

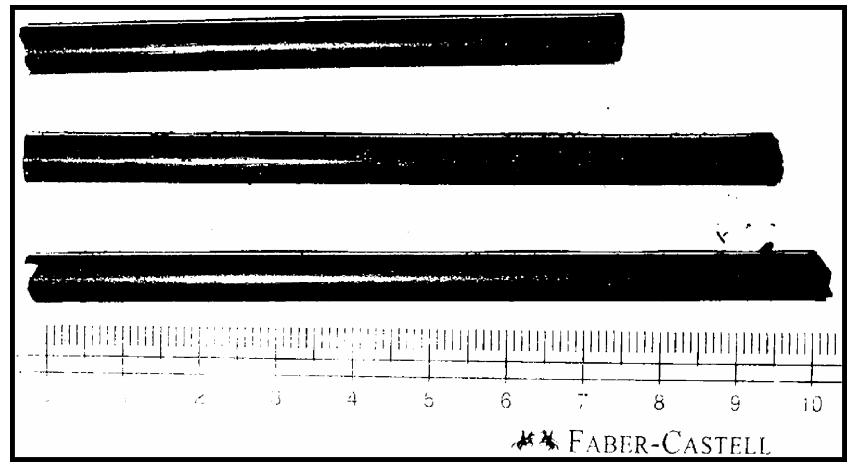

Figure 2. Optical image of the free-standing coaxial carbon cylinders.

\section{Results and discussion}

\subsection{Coaxial carbon cylinders consisting of aligned CNT stacks}

The as prepared coaxial carbon cylinders were found to have their lengths varying from $\sim 7-10 \mathrm{~cm}$ (figure 2). Surface morphologies of as prepared coaxial carbon cylinders were studied by SEM. Figure 3(a) shows the representative SEM image of the cross-section of periphery of carbon cylinder. The inset of figure 3(a) shows magnified view of a portion along the periphery. This image exhibits the CNT configuration consisting of two stacks in the coaxial carbon cylinder configuration. Figure 3(b) shows the formation of four stacks in the coaxial carbon cylinder configuration made after three interruptions of precursor flow. The CNT stacks consisted of aligned MWNTs in each case. 

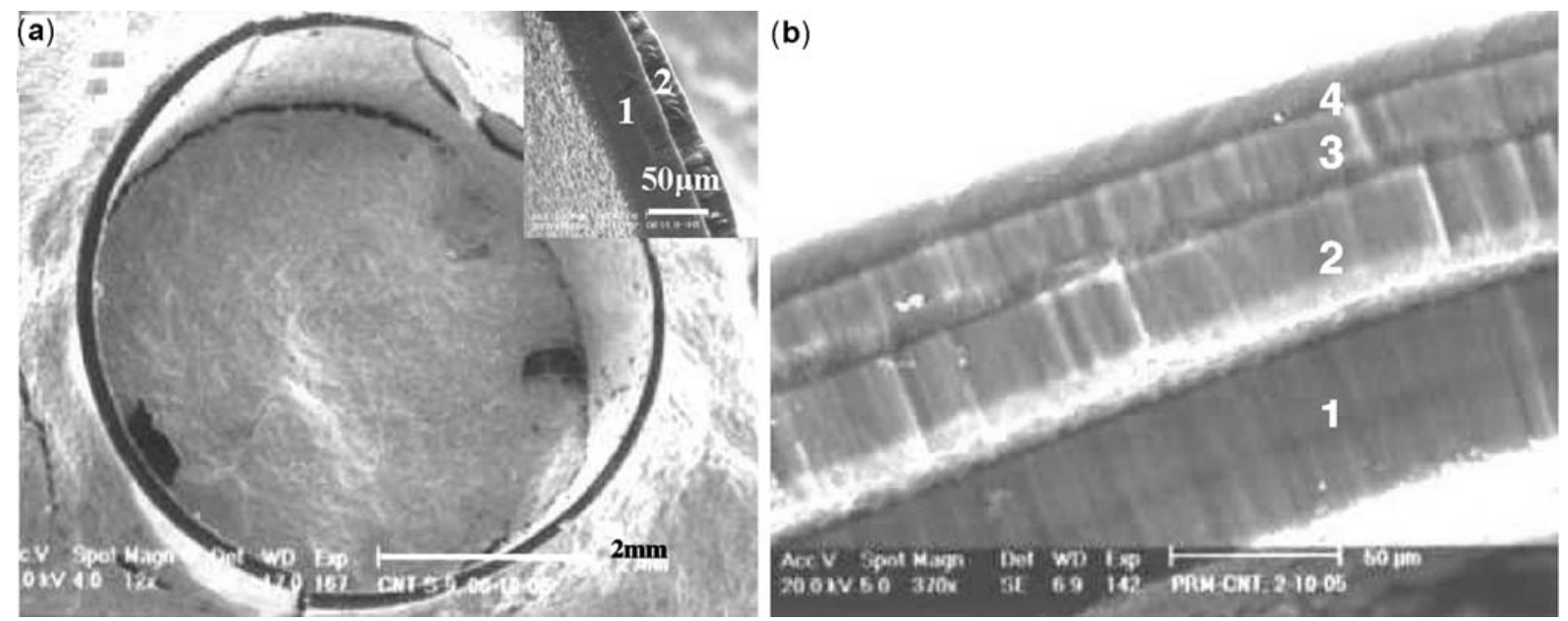

Figure 3. SEM images of cross-section of the two stacked carbon cylinders: (a) view of the periphery of two (inset of (a)) and four and (b) stacked carbon cylinders

The growth of carbon cylinder consisting of only one layer of radially aligned MWNTs has earlier been studied by us (Srivastava et al 2004). The benzene-ferrocene precursor flow with the carrier gas $\mathrm{Ar}$, Fe particles get deposited on the inner walls of the quartz tube through dissociation of ferrocene. Further supply of precursor allows dissociation of hydrocarbon on these precipitated Fe particles. The growth of MWNTs bundle, aligned radially, grows on the Fe particles forming the cylindrical configuration of CNTs. As regards the present study on the mode of growth of the second CNT stack, there are two possibilities. One possible route is that the pre-existing CNT stack may serve as template/substrate and the second CNT stack grows on top of this. This is depicted in figure 4 . The second possibility is that the precursor passes through the first CNT stack, reaches the base i.e. quartz tube and another CNT stack, similar to the first one which grows from the quartz tube base. If the first mechanism holds, the second CNT stack will be strongly bonded to the first one. However, it was found that the first CNT stack can be cleaved/peeled off easily from the second one. This suggests that the second stack did not grow on top of the first stack.

It is thus clear that the second CNT stack grows similar to the first stack from the quartz tube base. The precursor (benzene and ferrocene) and Ar gas pass through the first CNT stack and reach the base i.e. the quartz tube from where the CNT stack growth takes place. The first cylindrical stack is pushed up and sits on top of the now growing second CNT stack (figure 4). As regards the passing of the precursor and Ar vapours passing through the first cylindrical MWNT stack, it can take place via flow from the individual MWNT central core or through the interstitial spaces between MWNT and reach the quartz tube where the CNT bundles growth takes place. With regard to the length (width) of the CNT stack, it was found that the width corresponding to the length of the individual MWNT for the first stack is the largest $(\sim 55 \mu \mathrm{m}$ for the two stacks, $\sim 53 \mu \mathrm{m}$ for the four stacks). The width of the other successive CNT stacks is lower. We propose that the lowering of the width is due to lower precursor (benzene-ferrocene) flux reaching to base i.e. the quartz tube surface. When more than one CNT stack is present, the crossing of the precursor through flow paths (either through the central core of MWNT or the interstitial spaces between the nanotubes), may lead to a function of the precursor getting lost due to adsorption on the surface of first stack of MWNTs, condensation of the precursor before reaching the base or trapping of precursor in the tilted portions of the interstitial space.

\subsection{CNT-polymer composite films}

In order to explore the surface morphologies of MWNTsPEO composite, scanning electron microscopic studies were carried out. The SEM studies of MWNTs $(\sim 50 \mathrm{wt} \%)-$ PEO composite film show homogeneous dispersion of MWNTs in PEO matrix (inset of figure 5). The average diameter and length of MWNTs are $\sim 20-40 \mathrm{~nm}$ and $\sim 1-$ $5 \mu \mathrm{m}$, respectively. The CNTs appear interconnected at this high concentration and suggests a nanotube network.

The electrical transport property (d.c. electrical conductivity) of the MWNTs-PEO/PAM composite has been measured using the four-probe technique. The results of d.c. electrical conductivity for MWNTs-PEO composites at different MWNTs loading are summarized in table 1. The conductivity of film, in general, increases as the MWNTs concentration increases, which indicates that the electrical conduction across the film is mainly controlled by the nanotube network in polymer matrix.

In samples between 5 and $15 \mathrm{wt} \%$ the conductivity of composites is nearly constant, being around $10^{-8} \mathrm{~S} / \mathrm{cm}$. 

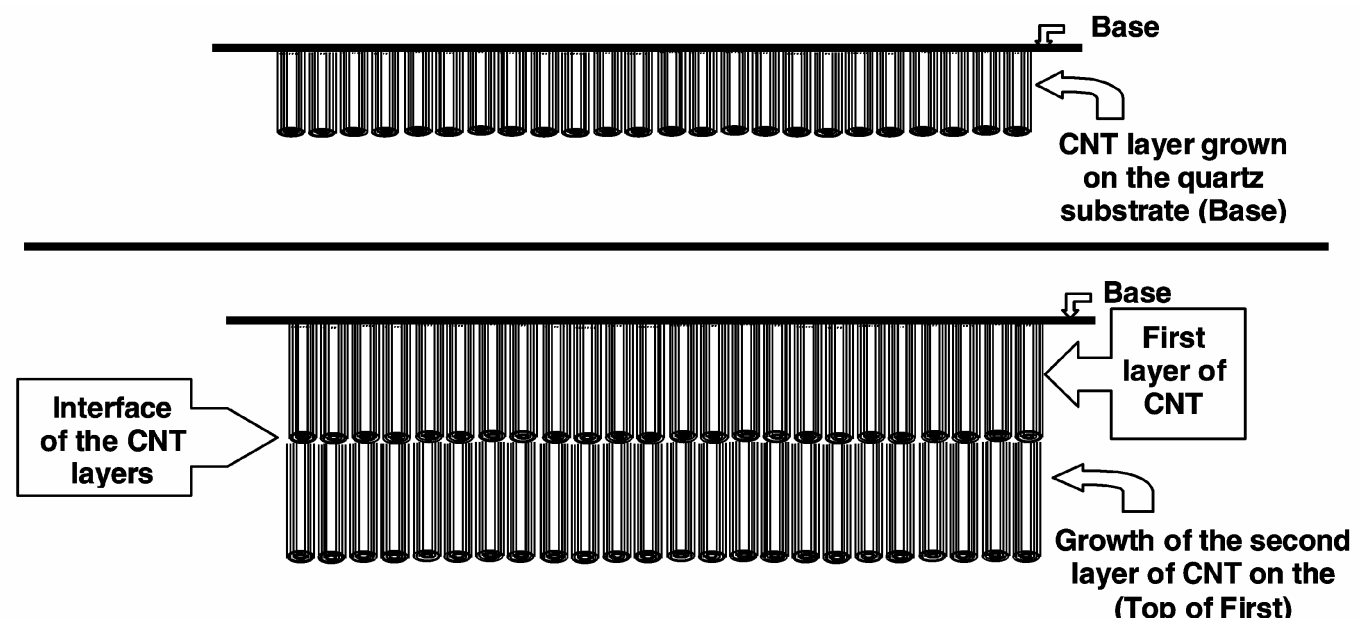

Mechanism I for the growth of CNTs stack (Possible Case)

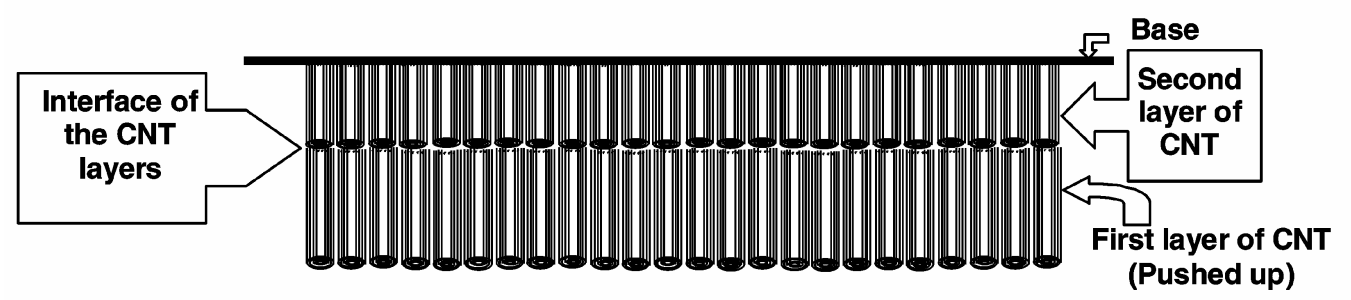

Mechanism II for the growth of CNTs stack (Present Case)

Figure 4. Tentative growth mechanism of CNT stacks over quartz substrate

Table 1. D.C. electrical conductivity data of MWNTs-PEO composites at room temperature.

\begin{tabular}{lcc}
\hline Sample & MWNTs loading (wt\%) & Conductivity $(\mathrm{S} / \mathrm{cm})$ \\
\hline PEO & 0 & $7.5 \times 10^{-8}$ \\
MWNTs-PEO & 5 & $8.5 \times 10^{-8}$ \\
& 10 & $9 \times 10^{-7}$ \\
& 15 & $9 \times 10^{-7}$ \\
& 20 & $7 \times 10^{-6}$ \\
& 30 & $8 \times 10^{-4}$ \\
& 40 & $3 \times 10^{-2}$ \\
& 50 & 6.52 \\
\hline
\end{tabular}

When MWNTs loading increases from 20-40 wt\%, the conductivity of composite increases from $\sim 10^{-6}-10^{-2} \mathrm{~S} / \mathrm{cm}$. This indicates that for a loading of MWNTs below $15 \mathrm{wt} \%$, the MWNTs are isolated with the electrical conductivity governed mainly by the characteristics of polymer. As the loading of MWNTs increases further, the average distance between the nanotubes becomes sufficiently small for the electrons to tunnel through the polymer or many physical contacts between nanotubes to be formed.

The temperature-dependent conductivity of the $\mathrm{PEO} /$ PAM and MWNTs-PEO/PAM composite films at different MWNTs concentrations has been studied from room temperature to $16 \mathrm{~K}$. The conductivity-temperature characteris- tics are shown in figure 5 for PEO and MWNTs ( $\sim 50 \mathrm{wt} \%)-$ PEO composite films. The measured conductivity of the PEO film is $\sim 7.5 \times 10^{-8} \mathrm{~S} / \mathrm{cm}$. The conductivity of PEO film does not change with temperature (curve (a) in figure 5).

In MWNTs $(\sim 50 \mathrm{wt} \%)-\mathrm{PEO}$ composite film, the conductivity increases as the temperature increases (curve (b) in figure 5), which shows the semiconducting behaviour. The conductivity is found to be $6.52 \mathrm{~S} / \mathrm{cm}$ at room temperature. Thus the conductivity of MWNTs-PEO composite increases by eight orders on increasing the concentration of MWNTs from 0 to $50 \mathrm{wt} \%$ in PEO polymer. For MWNTs-PAM composite film, the conductivity of the composite film increases by six orders of magnitude at $\sim 30 \mathrm{wt} \%$ of nanotube concentration.

The mechanical behaviour relating to elastic modulus and tensile strength of PEO and MWNTs-PEO composite films has been studied. Figure 6 compares the stressstrain curves of PEO and PEO with $50 \mathrm{wt} \% \mathrm{MWNTs}$. For PEO film the value of the elastic modulus obtained from curve 1 (figure 6) was $\sim 0.04 \mathrm{GPa}$ and the tensile strength was $\sim 0.5 \mathrm{MPa}$. As expected, the addition of CNTs has increased the modulus and strength of the PEO film. In MWNTs $(\sim 50 \mathrm{wt} \%)$-PEO composite film, the measured elastic modulus and tensile strength were $\sim 0.2 \mathrm{GPa}$ and $5 \mathrm{MPa}$ (curve 2 in figure 6$)$. Thus the MWNTs ( $\sim 50 \mathrm{wt} \%$ )PEO composite film is found to possess five times higher 
modulus than the PEO sample. The tensile strength of MWNTs $(\sim 50 \mathrm{wt} \%)-\mathrm{PEO}$ composite film is ten times higher than that of the PEO film.

We believe that the mechanical properties of the CNTpolymer composite are highly dependent on nanotube dispersion, which directly influences molecular tube-tube and tube-polymer interactions in the materials. Such molecular interactions will play a critical role in load transfer and interfacial bonding that determines mechanical properties of the nanocomposites. The variations in the CNT dispersion in the resultant composite could be the major reason for this phenomenon. For the PEO and MWNT ( $\sim 50 \mathrm{wt} \%$ )-PEO composite films, the behaviour of stress curves up to $\sim 1 \%$ strain is different. From figure 6 , curve

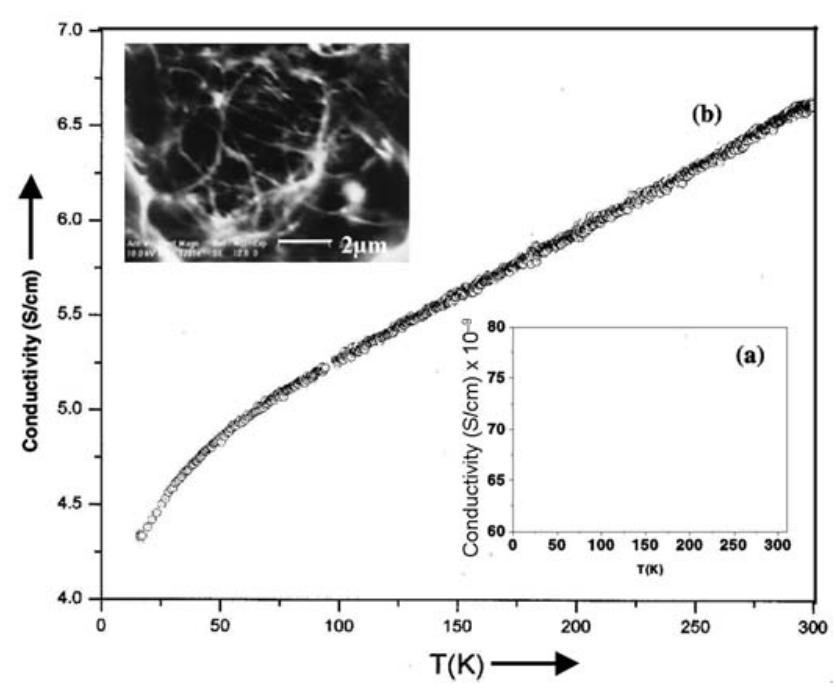

Figure 5. Conductivity vs temperature curves for (a) $\mathrm{PEO}$ and (b) MWNTs $(\sim 50 \mathrm{wt} \%)-\mathrm{PEO}$ composite films. The inset is SEM micrograph of MWNTs ( $50 \mathrm{wt} \%)$ - PEO composite.

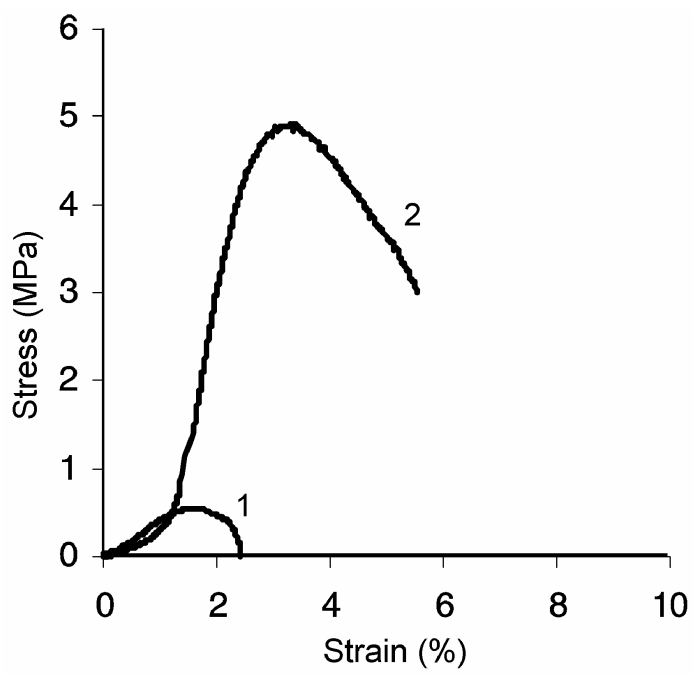

Figure 6. Stress-strain curves of PEO (curve 1) and MWNT ( $\sim 50 \mathrm{wt} \%)$-PEO (curve 2) composite films.
2 has smaller initial stress-strain slope than curve 1 . In this case, it appears that the alignment effect of CNTs plays a large role for getting different onset behaviour. At high MWNT concentrations, the random motion of CNTs is impeded, but alignment can easily be achieved. Therefore, in a sample with high MWNT concentration, it is easier to get MWNTs aligned with each other than with low concentration of nanotubes. This in the end contributes to the much higher modulus (from $\sim 1 \%$ strain on). The stress of the MWNT ( $50 \mathrm{wt} \%)-\mathrm{PEO}$ film increases rapidly when the strain exceeds about $1 \%$ (curve 2). At $3 \%$ strain, the stress of the composite is maximum. On the other hand, up to this point $(\sim 1 \%$ strain), where the alignment is taking place, it seems that pure PEO is better. For the PEO film, the stress is maximum at $\sim 1.5 \%$ strain. The mechanical behaviour of PAM and MWNTsPAM composite films are being carried out and the results will be forthcoming.

\section{Conclusions}

Based on the present investigations, the following conclusions can be drawn:

(I) The coaxial carbon cylinders consisting of aligned CNT stacks have been prepared by controlled spray pyrolysis technique employing benzene-ferrocene $(\sim 30 \mathrm{mg} / \mathrm{ml})$ precursor and Ar as carrier gas. The coaxial carbon cylinders consisting of aligned CNTs have been prepared without using any patterned substrates. The CNT stacks have been produced by periodically interrupting the flow of precursor.

(II) The MWNTs-polymer (PEO/PAM) composite films have been prepared by solution cast technique and then these have been characterized by employing the SEM technique. The conductivity measurements on the MWNTsPEO composite films with highest concentration of MWNTs $(\sim 50 \mathrm{wt} \%)$ showed an increase of eight orders $\left(\sim 7.5 \times 10^{-8}-6.52 \mathrm{~S} / \mathrm{cm}\right)$ of magnitude in conductivity from bare PEO film. The temperature dependence of the conductivity for MWNTs ( $\sim 50 \mathrm{wt} \%)-\mathrm{PEO}$ composite showed predominantly semiconducting behaviour. For MWNTs ( $\sim 50 \mathrm{wt} \%)$-PEO composite film, tensile tests revealed five times increase in elastic modulus and ten times increase in tensile strength as compared to the PEO film. With respect to pure PAM, the conductivity of MWNTs ( $30 \mathrm{wt} \%)$-PAM composite film increases by six orders of magnitude.

\section{Acknowledgements}

The authors are grateful to the Nano Science and Technology Initiative, Department of Science and Technology (DST), India for financial support. We would also like to thank Prof. C N R Rao, Prof. A K Raychoudhary, Prof. A K Sood, Prof. Panjab Singh, and Prof. S Lele. The fi- 
nancial support from CSIR and MNRE, New Delhi, are also gratefully acknowledged.

\section{References}

Awasthi K, Singh A K and Srivastava O N 2003 J. Nanosci. Nanotechnol. 3540

Awasthi K, Awasthi S, Srivastava A, Kamalakaran R, Talapatra S, Ajayan P M and Srivastava O N 2006 Nanotechnology 17 5417

Ebbsen T W and Ajayan P M 1992 Nature 258220

Grossiord N, Loos J, Regev O and Koning C E 2006 Chem. Mater. 181089

Iijima S 1991 Nature 35456

Jia Z J et al 1999 Mater. Sci. Eng. A271 395

Jin J X, Pramoda K P, Goh S H and Xu G Q 2002 Mater. Res. Bull. 37271

Kymakis E, Alexandou I and Amaratunga G A J 2002 Synth. Metals 12759
Li X, Cao A, Jung Y J, Vajtai R and Ajayan P M 2005 Nanoletters 51997

Meyyappan M 2005 (Boca Raton, FL: CRC Press) p. 1

Mishra P R, Awasthi K and Srivastava O N 2007 J. Nanosci. Nanotechnol. 71815

Moniruzzaman M and Winey K I 2006 Macromolecules 39 5194

Singh C, Shaffer M S P and Windle A H 2003 Carbon 41359

Srivastava A, Srivastava O N, Talapatra S, Vajtai R and Ajayan P M 2004 Nature Mater. 3610

Thess A, Lee R, Nikolaev P, Dai H J, Petit P and Robert J 1996 Science 273483

Vajtai R, Wei B Q, Zhang Z L, Jung Y, Ramanath G and Ajayan P M 2002 Smart Mater. Struct. 11691

Vivekchand S R C, Cele L M, Deepak F L, Raju A R and Govindraj A 2004 Chem. Phys. Letts 386313

Zhang Z J, Wei B Q, Ramanath G and Ajayan P M 2000 Appl. Phys. Lett. 773764

Zhu L, Xiu Y, Hess D W and Wang C P 2005 Nanoletters 5 24613 